\title{
In-Vitro and In-Vivo Pathogenicity Study of Cryptococcus Species
}

\author{
A. Banerjee ${ }^{1}$, S. Pradhan ${ }^{1 *}$, C. Debnath ${ }^{2}$, S. Baidya ${ }^{3}$, N.C. Patra ${ }^{1}$, R.N. Hansda ${ }^{1}$, \\ S. Mondal ${ }^{1}$ and S.K. Mukhopadhayay ${ }^{1}$ \\ ${ }^{1}$ Department of Veterinary Pathology, ${ }^{2}$ Department of Veterinary Public Health, ${ }^{3}$ Department of \\ Veterinary Parasitology, Faculty of Veterinary and Animal Sciences, \\ WBUAFS, Kolkata-700037, India \\ *Corresponding author
}

A B S T R A C T

Ke y w o r d s
$\begin{aligned} & \text { Patho-physiology, } \\ & \text { RAPD, Genetic } \\ & \text { variability, } \\ & \text { Pathogenicity. }\end{aligned}$
Article Info
$\begin{aligned} & \text { Accepted: } \\ & 19 \text { October } 2017 \\ & \text { Available Online: } \\ & 10 \text { December } 2017\end{aligned}$

Cryptococcus neoformans encapsulated yeast causes localized or disseminated infections, which may be acute, sub-acute or chronic in nature, in immunocompromized and apparently immunocompetent patients. Unfortunately, knowledge concerning the genetic variability in relation to the pathophysiology of the disease remains controversial. Random Amplification of Polymorphic DNA (RAPD) technique is a novel molecular approach for detection of genetic variability of fungal strains. A RAPD technique using arbitrary primers R28, OPH-02 and OPH-20 clearly indicates genetic variability in C. neoformans. To find out relationship between genetic variability and pathogenicity of the $C$. neoformans, we carried out the in- vitro and in-vivo pathogenicity test in mice model, infected with four different RAPD types of Cryptococci. Mice showed a high susceptibility to Pattern I and Pattern IV of C. neoformans. This variation in susceptibility is associated with some minute difference in their genotypes but not related with the in-vitro virulence factors i.e. urease activity and melanin production.

\section{Introduction}

The member of Cryptococcus neoformans species complex, Cryptococcus neoformans and Cryptococcus gattii are the cause of cryptococcosis of human and animal (Buchanan and Murphy, 1998; Kwon-Chung et al., 2002). Among these, Cryptococcus neoformans has been increasingly reported from immunocompromised patients. This encapsulated yeast causes localized or disseminated infections, which may be acute, sub-acute or chronic in nature, in both immunocompromised and apparently immunocompetent patients (Kwon-Chung and Bennett, 1992). The spectrum of predisposing immunosuppression includes connective tissue disorders, malignancies, diabetes mellitus, steroid-dependent pulmonary disorders, immunosuppressive regimens in relation to transplants, and human immunodeficiency virus (HIV)/Acquired immune deficiency syndrome (AIDS).

India has the second largest burden of Cryptococcosis due to an estimated population of 3.1 million to 9.4 million person living with HIV/AIDS (UNAIDS. 2006). The country has a documented high prevalence $(1.7-4.7 \%)$ of cryptococcosis in the person 
with HIV/AIDS (Kumarasamy et al., 2003; Vajpayee et al., 2003)

Yamamoto et al., (1995) have developed a random amplification of polymorphic DNA (RAPD) techniques using arbitrary primers R28, OPH-02 and OPH-20, which clearly indicates genetic variability in $C$. neoformans (Yamamoto et al., 1995). In this study we compare pathogenicity of RAPD types in both in-vitro and in-vivo model to find out relationship between genetic variability and pathogenicity of organism.

\section{Materials and Methods}

\section{Source of the samples}

RAPD typing of environmental isolates had already conducted by the Outreach Programme on Zoonotic Diseases (OPZD) at Department of Veterinary Public Health of West Bengal University of Animal and Fishery Sciences. 4 distinct RAPD fingerprint patterns (designated as pattern-I through pattern-IV) were identified by 3 primers R-28, $\mathrm{OPH}-02$ and $\mathrm{OPH}-20$ and preserved in $-80^{\circ} \mathrm{C}$. Representative strains of each RAPD types were used for pathogenicity study (Fig. 1).

\section{In-vitro pathogenicity study}

\section{Biochemical tests}

Isolates were also inoculated on C.G.B. (Canavanine- Glycine- Bromothymol Blue) agar media at $25-30^{\circ} \mathrm{C}$ up to 5 days to differentiate $C$. neoformans and $C$. gattii. In case of $C$. neoformans var. neoformans, colour would not change (Kwon-Chung et al., 1982).

\section{Urease activity}

This test was performed in Christensen's urea agar slant which was inoculated with the test fungus and incubated at $28^{\circ} \mathrm{C}$ for 7 days. The urease activity usually within 7 days changes the colour of the medium to pink (Cox et al., 2000).

\section{Melanin formation in Bird Seed Agar}

Isolates were cultured in Bird Seed Agar $\left(\right.$ Himedia $\left.{ }^{\circledR}\right)$. The plates were incubated at $28^{\circ} \mathrm{C}$ for 2 days and watched for brown pigmentation. All the four RAPD Types are inoculated in Bird Seed Agar (BSA) for the study of Melanin formation.

\section{In-vivo pathogenicity study}

Animal experiments were conducted with the approval of Institutional Animal Ethics Committee [IAEC/93/VI(M)] and Institutional Bio-safety Committee [10/IBC/VAS/17] of West Bengal University of Animal and Fishery Science.50 Swiss male Albino mice with average body weight $25 \mathrm{gm}$ divided in five groups for each RAPD types, CN1 (Pattern I), CN2 (Pattern II), CN5 (Pattern III) and CN9 (Pattern IV) and the last group was used for control and each group having 10 animals. After overnight culture on brain heart infusion broth at $28^{\circ} \mathrm{C}$, yeast cells of $C$. neoformans surrounded by intermediate capsule were harvested and then suspended in sterilized Phosphate Buffer Saline (PBS). Infective inoculum was prepared by adjusting number of yeast cells $3.25 \times 10^{5} / \mathrm{ml}$ measured in a hemocytometer. Each mouse was intravenously inoculated $0.2 \mathrm{ml}$ of inoculum into lateral tail vein. 2 mice from each group were sacrificed after $7^{\text {th }}, 14^{\text {th }}, 21^{\text {st }}$ and $28^{\text {th }}$ Day of post infection. Mice were euthanized with ketamine @ 200mg/kg b.w. intraperitoneally and P.M. changes were recorded. The tissues thus collected were kept in vials containing $10 \%$ neutral buffered formalin for histopathological examination. After processing tissue samples were sectioned at5 micrometer and stained by Hematoxylin and 
Eosin (Suvarna et al., 2012). Quantitative assessment of the fungal infection area was performed by observing 10 selected fields randomly at $\mathrm{x} 400$ magnification, with the following criteria: 0 , no fungi; + , area of fungal infection $<25 \%$; ++ , infected area involving $25-50 \%$ of the field; and +++ , infected area $\geq 50 \%$ of the field (Surawut et al., 2017).

\section{Results and Discussion}

\section{In-vitro pathogenicity study}

\section{Biochemical test}

All the isolates gave negative test on Canavanine- Glycine-Bromothymol Blue (CGB) Agar (Fig. 2), indicating that isolates were $C$. neoformans var. neoformans.

\section{Urease}

All RAPD Types were urease positive in Christensen's medium and changed colour of the medium from yellow to bright pink (Fig. 3).

\section{Melanin production}

Melanin production was assessed by verifying the extent of brown pigment formation was confirmed in all the strains. Melanin production did not differ significantly among the all RAPD types (Fig. 4; Table 1).

\section{In-vivo pathogenicity study}

\section{Clinical signs}

Mice showed clinical signs of disease like hunching, panting, weight loss, reduced food/water intake and inactivity between day 7 and day 9 post infections.

\section{Gross morphology and necropsy finding}

No abnormality was detected when mice were sacrificed after one week of inoculation. But Carcass was severely dehydrated, emaciated. Doming/Enlargement of head were observed 14 days after inoculation (Fig. 5). Sever congestion of meninges and nodular opaque regions were detected in kidney after two and three weeks of inoculation (Fig. 6).

\section{Histopathological studies}

Histopathologically, the brain and liver were severely affected with the fungus. In the brain cysts with Cryptococci continued to increase until the end of the experiment (Fig. 7 and 8). On the other hand, numerous mononuclear cells infiltrate into the live and produced several foci of granulomatous lesion with Cryptococci after 14 days of inoculation (Fig. 9 and 10). Quantitative measurement of the pathogenicity were performed by observing cyst and granuloma formation in liver and brain on $7^{\text {th }}, 14^{\text {th }}, 21^{\text {st }}$ and $28^{\text {th }}$ days. The majority of these strains were environmental isolates. This study revealed that $\mathrm{CN} 1$ and CN9 both of the isolates produced marked degree of cyst and granuloma formation in brain, liver (Table 2).

The frequency and mortality due to opportunistic mycoses are increasing among patients with AIDS, hematologic malignancies, and transplants. This emphasized the importance of understanding the pathophysiology of this emerging fungal pathogen. Unfortunately, knowledge concerning the genetic variability in relation to the pathophysiology of the disease remains controversial. RAPD technique is a novel molecular approach for detection of genetic variability of fungal strains. 
Table.1 RAPD fingerprint pattern of the 14 isolated $C$. neoformans strains. (Kindly supplied by Outreach Programme on Zoonotic Diseases, Department of Veterinary Public Health, WBUAFS)

\begin{tabular}{|c|c|c|c|c|}
\hline Strain & & RAPD p & & \\
\hline no. & Primer OPH-20(A) & Primer OPH-02(B) & Primer R-28(C) & Pattern \\
\hline CN1 & $\mathrm{a}$ & $\mathrm{i}$ & 1 & I \\
\hline $\mathrm{CN} 2$ & b & ii & 2 & II \\
\hline $\mathrm{CN} 3$ & a & $\mathrm{i}$ & 1 & I \\
\hline CN4 & c & iii & 3 & III \\
\hline CN5 & $\mathrm{c}$ & iii & 3 & III \\
\hline CN6 & b & ii & 2 & II \\
\hline CN7 & - & - & 3 & IV \\
\hline $\mathrm{CN} 8$ & $\mathrm{a}$ & $\mathrm{i}$ & 1 & I \\
\hline CN9 & - & - & 3 & IV \\
\hline CN10 & b & ii & 2 & II \\
\hline CN11 & - & - & 3 & IV \\
\hline CN12 & $\mathrm{a}$ & $\mathrm{i}$ & 1 & I \\
\hline CN13 & b & ii & 2 & II \\
\hline CN14 & $\mathrm{a}$ & $\mathrm{i}$ & 1 & I \\
\hline
\end{tabular}

Table.2 Quantitative assessment of Cryptococcal infection in brain and liver X400

\begin{tabular}{|l|l|c|c|c|c|}
\hline \multicolumn{2}{|r}{ Days } & $7^{\text {th }}$ day & $14^{\text {th }}$ day & $21^{\text {st }}$ Day & $28^{\text {th }}$ Day \\
\hline \multirow{2}{*}{ CN1 } & Brain & 0 & + & ++ & +++ \\
\cline { 2 - 6 } & Liver & 0 & + & +++ & +++ \\
\hline \multirow{2}{*}{ CN2 } & Brain & 0 & + & + & + \\
\cline { 2 - 6 } & Liver & 0 & + & + & + \\
\hline \multirow{2}{*}{ CN5 } & Brain & 0 & + & + & + \\
\cline { 2 - 6 } & Liver & 0 & + & + & + \\
\hline \multirow{2}{*}{ CN9 } & Brain & 0 & + & ++ & +++ \\
\cline { 2 - 6 } & Liver & 0 & + & ++ & +++ \\
\hline
\end{tabular}


Fig.1 Primer OPH-20 revealed major differences in banding patterns ranging between 1500bp and $400 \mathrm{bp}$, which distinguished the profiles a, b and c. Primer OPH-02 revealed 3 different profiles in banding patterns ranging between about ranging between about 2000bp and 400bp, which distinguished the profiles in three categories namely i, ii and iii. Primer R-28 revealed 3 different profiles in banding pattern ranging between 1500bp and 200bp, which distinguished the profiles in 1,2 and 3 categories. (Kindly supplied by Outreach Programme on Zoonotic Diseases, Department of Veterinary Public Health, WBUAFS)

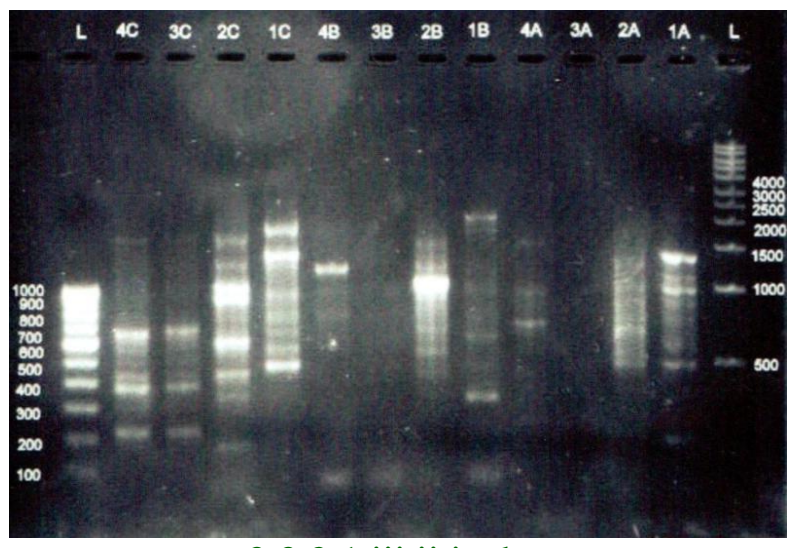

3321 iii ii i c b a

$\mathrm{R}-28(\mathrm{C}) \mathrm{OPH} 02(\mathrm{~B}) \mathrm{OPH} 20(\mathrm{~A})$

Fig.2 Growth on CGB agar. Showing colonies of C. neoformans with no colourchange of media(left side) and uninoculated control (right side)

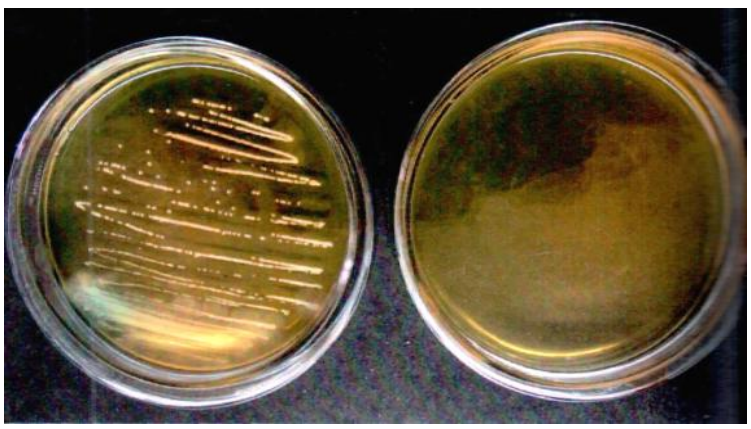

Fig.3 Urease Activity of $C$. neoformans. First tube from left side used as control and other tubes showing positive Urease activity by developing pink colour

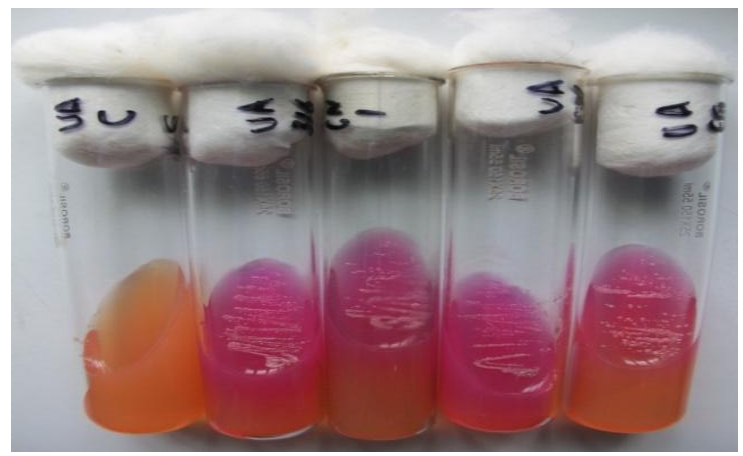


Fig.4 Brown coloured colonies of C. neoformans CN9 in Bird Seed Agar

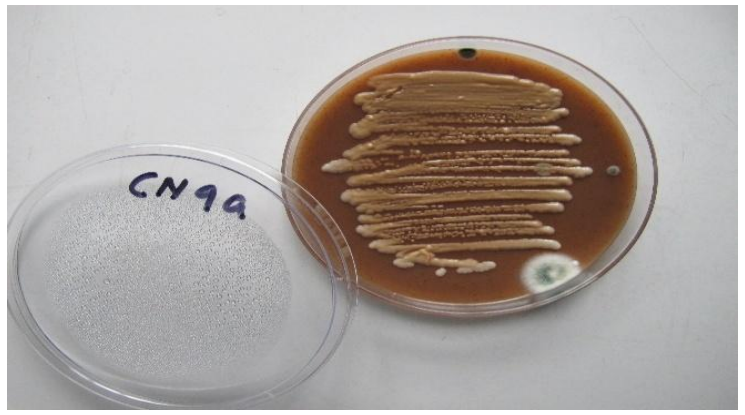

Fig.5 Mice showing marked degree of chacexia and doming of head 14 days after Incoulation of C. neoformans

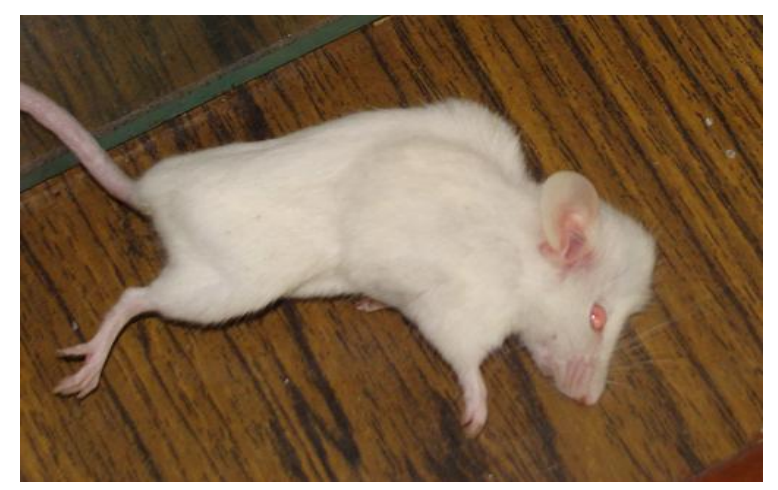

Fig.6 Marked degree of congestion of meninges 14 days after inoculation of C. neoformans

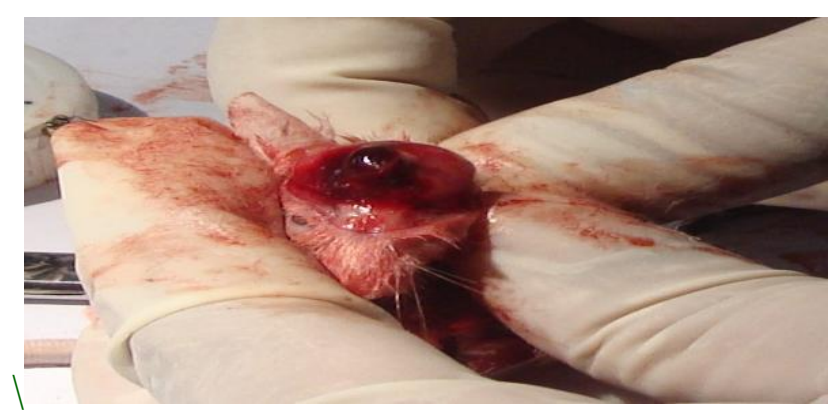

Fig.7 Cyst formation in brain 14 days after inoculation. H and E. X100

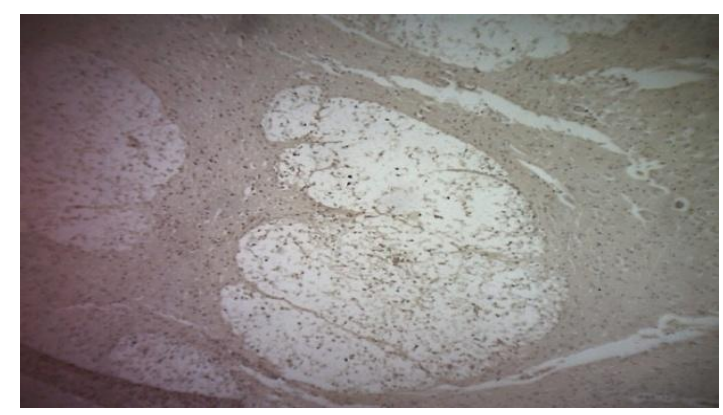


Fig.8 Cyst containing numerous yeast cells(arrow) in the brain 14 days after inoculation of $3.25 \times 10^{5}$ cryptococci. Sothgate's mucicarmine stain, X400

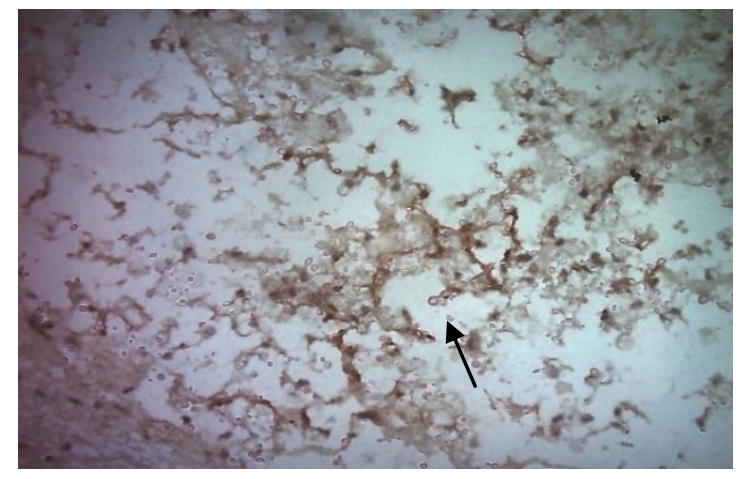

Fig.9 Granuloma(arrow) formation in liver, H andE, X100

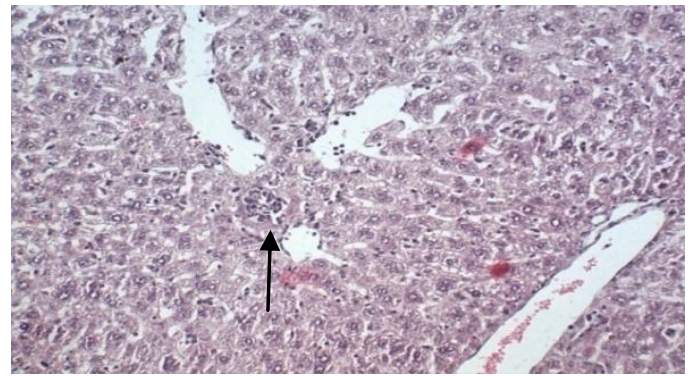

Fig.10 Granuloma in Liver 14 days after inoculation, HandE, X400

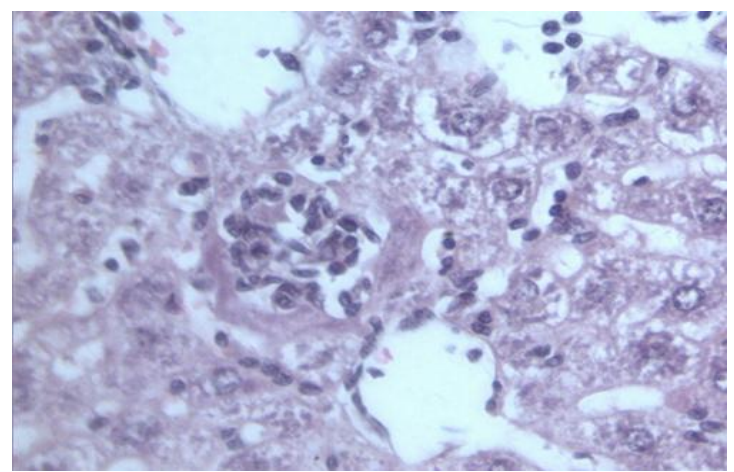

To elucidate this of the $C$. neoformans, we investigated the biological characteristics of pathogens and the pathophysiology of mice infected with four different RAPD types of Cryptococci. Urease catalyzes the hydrolysis of urea to ammonia and carbamate. Under physiological conditions, this reaction can result in an increase in $\mathrm{pH}$. Urease activity has been found in several bacteria, fungi, and plants and has been shown to be an important pathogenic factor (Eaton et al., 1991). Melanin was found to be highly effective in protecting fungal structures from lysis or decomposition by natural communities of microorganisms (Kuo and Alexander, 1967).

In vitro results of urease activity, melanin production showed that all the tested RAPD types expressed these virulence factors, although to different extents. In the present 
histopathological study $\mathrm{CN} 1$ and $\mathrm{CN} 9$ showed a higher degree of pathogenicity and also provided evidence again for the important role of cell-mediated immunity to experimental cryptococcosis. In the liver, yeast cells inoculated intravenously into mice were engulfed by macrophages of the organ as soon as carried to it by blood stream. Miyaji and Nishimura suggested that these macrophages might not have a fungicidal ability against them. In order to destroy them the cell-mediated immunity had to be exerted (Miyaji and Nishimura, 1981). In the liver granuloma was the specific lesion of cellmediated immunity induced by $C$. neoformans and yeast cells were destroyed in the granulomatous tissue. This study also suggested that major histopathological features of the central nervous system were cystic and cysts with cryptococci expanded and continued to increase until the end of the experiment. In this study the number of granulomas and cryptococci reached a peak on the 14th day in the mice and thereafter decreased, except CN1 (Pattern1) and CN9(Pattern IV), which produced significantly higher level of infection in liver and brain even after 28 days.

Overall, these findings suggest that RAPD types belong to isolates $C$. neoformans have different pathogenicity in murine model. Mice showed a high susceptibility to Pattern I and Pattern IV of $C$. neoformans. This variability in susceptibility is associated with some minute difference in their genotypes but not related with the in-vitro virulence factors i.e. urease activity and melanin production.

\section{Acknowledgement}

Authors are grateful to Vice-Chancellor, West Bengal University of Animal and Fishery Sciences; Dean, Faculty of Veterinary and Animal Sciences, WBUAFS and concerned Heads of the Department of Veterinary Public
Health, Department of Veterinary Pathology, Department of Veterinary Parasitology, WBUAFS, Kolkata, India for providing necessary facilities to do research work.

\section{References}

Buchanan, K. L., and Murphy, J. W. (1998). What makes Cryptococcus neoformans a pathogen? Emerging infectious diseases, 4(1), 71.

Cox, G. M., Mukherjee, J., Cole, G. T., Casadevall, A., and Perfect, J. R. (2000). Urease as a virulence factor in experimental cryptococcosis. Infection and immunity, 68(2), 443-448.

Eaton, K. A., Brooks, C. L., Morgan, D. R., and Krakowka, S. (1991). Essential role of urease in pathogenesis of gastritis induced by Helicobacter pylori in gnotobiotic piglets. Infection and immunity, 59(7), 2470-247.

Kumarasamy, N., Solomon, S., Flanigan, T. P., Hemalatha, R., Thyagarajan, S. P., and Mayer, K. H. (2003). Natural history of human immunodeficiency virus disease in southern India. Clinical Infectious Diseases, 36(1), 79-85.

Kuo, M. J., and Alexander, M. (1967). Inhibition of the lysis of fungi by melanins. Journal of Bacteriology, 94(3), 624-629.

Kwon-Chung, K. J., Boekhout, T., Fell, J. W., and Diaz, M. (2002). (1557) Proposal to conserve the name Cryptococcus gattii against $C$. hondurianus and $C$. bacillisporus (Basidiomycota, Hymenomycetes, Tremellomycetidae). Taxon, 51(4), 804-806.

Kwon-Chung, K. J., Polacheck, I. T. Z. H. A. C. K., and Bennett, J. E. (1982). Improved diagnostic medium for separation of Cryptococcus neoformans var. neoformans (serotypes A and D) and Cryptococcus neoformans var. 
gattii (serotypes B and C). Journal of Clinical Microbiology, 15(3), 535-537

Kwon-Chung, K.J. and Bennett, J.E. (1992). Medical Mycology, Philadelphia:Lee and Febiger, Pp. 397-445.

Miyaji, M., and Nishimura, K. (1981). Studies on organ specificity in experimental murine cryptococcosis. Mycopathologia, 76(3), 145-154.

Surawut, S., Ondee, T., Taratummarat, S., Palaga, T., Pisitkun, P., Chindamporn, A., and Leelahavanichkul, A. (2017). The role of macrophages in the susceptibility of Fc gamma receptor IIb deficient mice to Cryptococcus neoformans. Scientific reports, 7, 40006.

Suvarna, K. S., Layton, C., and Bancroft, J. D. (2012). Bancroft's Theory and
Practice of Histological Techniques EBook. Elsevier Health Sciences.

UNAIDS. (2006). Report on the Global AIDS Epidemic [Online] Available at: http://data.unaids.org/pub/Global Report/ 2006/2006_gr_ch02_en.pdf. Accessed on 4 May 2011.

Vajpayee, M., Kanswal, S., Seth, P., and Wig, N. (2003). Spectrum of opportunistic infections and profile of CD4+ counts among AIDS patients in North India. Infection, 31(5), 336-340.

Yamamoto, Y., Kohno, S., Koga, H., Kakeya, H., Tomono, K., Kaku, M. and Hara, K. (1995). Random amplified polymorphic DNA analysis of clinically and environmentally isolated Cryptococcus neoformans in Nagasaki. Journal of Clinical Microbiology, 33(12), 33283332.

\section{How to cite this article:}

Banerjee, A., S. Pradhan, C. Debnath, S. Baidya, N.C. Patra, R.N. Hansda, S. Mondal and Mukhopadhayay, S.K. 2017. In-Vitro and In-Vivo Pathogenicity Study of Cryptococcus Species. Int.J.Curr.Microbiol.App.Sci. 6(12): 2495-2503. doi: https://doi.org/10.20546/ijcmas.2017.612.290 